Research Article

\title{
Field Localization and Density Cavitation in Low-Beta Plasmas
}

\author{
Motilal Rinawa $\mathbb{D}^{1},{ }^{1}$ Prashant Chauhan $\mathbb{D}^{2},{ }^{2}$ Sintu Kumar ${ }^{(D)},{ }^{3}$ Manoj Kumar Singh $\left(\mathbb{D},{ }^{4}\right.$ \\ Hari Kumar Singh $\left(\mathbb{C}^{5}\right.$, Amit Sharma $\oplus^{6},{ }^{6}$ and R. P. Sharma $\oplus^{1}$ \\ ${ }^{1}$ Centre for Energy Studies, Indian Institute of Technology Delhi, Delhi 110016, India \\ ${ }^{2}$ Department of Physics and Material Science and Engineering, Jaypee Institute of Information Technology, Noidar, \\ Uttar Pradesh, India \\ ${ }^{3}$ Department of Physics, DeenDayal Upadhyay Gorakhpur University, Gorakhpur-273009, Uttar Pradesh, India \\ ${ }^{4}$ Department of Mechanical Engineering, Faculty of Engineering and Technology, MJP Rohilkhand University, Bareilly, \\ Uttar Pradesh, India \\ ${ }^{5}$ Electronics and Communication Engineering, M. J. P. Rohilkhand University, Bareilly, Uttar Pradesh, India \\ ${ }^{6}$ Department of Physics, D.A.V. (PG) College, Dehradun, India
}

Correspondence should be addressed to Prashant Chauhan; prashant.chauhan@jiit.ac.in

Received 28 May 2021; Revised 1 November 2021; Accepted 9 November 2021; Published 28 November 2021

Academic Editor: Yongtao Zhao

Copyright $\odot 2021$ Motilal Rinawa et al. This is an open access article distributed under the Creative Commons Attribution License, which permits unrestricted use, distribution, and reproduction in any medium, provided the original work is properly cited.

\begin{abstract}
In the present paper, filamentous structure formation, associated turbulent spectrum, and density cavity formation phenomena have been investigated for low- $\beta$ plasma $\left(\beta \ll m_{e} / m_{i}\right)$ applicable to the auroral region. A set of dimensionless equations governing the dynamics of three dimensionally propagating inertial Alfvén wave (3D-IAW) and perpendicularly propagating magnetosonic wave (PMSW) has been developed. Ponderomotive force due to 3D-IAW has been included in the dynamics of the PMSW. Numerical simulation has been performed to study the nonlinear coupling of these two waves. From the obtained results, we found that the field intensity localization takes place which may further lead to the additional dissipation/turbulence process for particle heating and acceleration in space plasma. The associated turbulent spectrum is obtained with scaling nearly $k^{-4.28}$ at smaller scales (in the dissipation range). Relevance of the obtained results with the observations reported by various spacecrafts such as Hawkeye and Heos 2 has been discussed. Also, density fluctuations (depletion) of $\sim 0.10 n_{0}$ are calculated, which are consistent with the FAST spacecraft observation reported.
\end{abstract}

\section{Introduction}

Alfvén waves (AWs) were first theoretically predicted by Hannes Alfvén in 1942 and later verified experimentally by Bostick and Levine in [1]. These are the low-frequency electromagnetic waves propagating along the background magnetic field in space and laboratory plasmas $[2,3]$. These waves are nondispersive in nature but can obtain dispersive properties due to finite frequency correction and finite electron inertia effects [4] in low- $\beta$ plasmas with $\beta \ll m_{e} / m_{i}$ (wherem $m_{e} / m_{i}$ is the ratio of the electron mass to the ion mass and $\beta$ is the ratio of the thermal pressure to the magnetic pressure). When their perpendicular length scales become finite and comparable to the electron inertial length [5] or to the ion gyroradius/finite gyroradius, these waves become dispersive in nature and are known as dispersive Alfvén waves (DAWs). The most vital physical property of the DAWs is the presence of the finite parallel electric field [6]. Inertial Alfvén waves (IAWs) are known to play a vital role in the energization of the auroral plasma $[7,8]$.

In the literature, several authors have studied the strong density depletion in the low- $\beta$ plasma. Wu et al. [9] discussed the density depletion in the corona due to the presence of beams of energetic particles and magnetic compression.

Many researchers $[10,11]$ have pointed out the role of solitary IAWs, which are accompanied by both density dips and humps in auroral particle heating. In the literature, it has been suggested that these cavities are associated with the ponderomotive nonlinearity of the inertial Alfvén wave [12]. 
The nonlinear evolution of IAWs and magnetosonic waves (MSWs) taking nonadiabatic response of the magnetic field for the low- $\beta$ plasmas is carried out by many researchers. These cavities are known to be associated with low-frequency electromagnetic perturbations and have perpendicular width of the order of the electron skin depth, i.e., $c / \omega_{p e}[13-15]$.

Observational signatures of the presence of AWs in auroral regions of Earth's ionosphere are also reported in the literature $[16,17]$. Several observed phenomena in the space plasmas are known to be associated with the nonlinear DAWs. Many authors have studied various mechanisms of electron acceleration and ion heating through DAWs. Hasegawa and Chen [18] discussed the utilization of Alfvén waves in the heating of plasma particles. Stochastic ion heating by the decay of the DAW was reported by Stasiewicz et al. [19]. Besides Hasegawa and Stasiewicz, other researchers also studied different mechanisms of particle acceleration such as modulation instabilities [20], turbulent dissipation [21-23], and field localization [24]. Zhao et al. $[25,26]$ also studied nonlinear interaction between kinetic Alfvén waves (KAWs) and the electrostatic and magnetostatic convective cells in plasmas. The formation of coherent structures in auroral plasmas is governed by the nonlinear dispersion relation and interaction of KAW and convective cells. The localized structures thus obtained offer a possible mechanism for the dissipation and offer a possible way of energy transportation from larger scales to the smaller scales.

The nonlinear coupling between IAWs and MSWs, taking ponderomotive force into account, has also been investigated in a great deal in the literature which plays a crucial role for the particle acceleration and plasma heating in the space plasma. Kumar and Singh [27] examined the nonlinear interplay between the low-frequency IAW and magnetosonic wave for the auroral region. They found that the turbulence spectrum follows the power law $\left(\sim k^{-5 / 3}\right)$ at a larger scale (in the inertial range) using numerical simulation techniques. Magnetic field turbulence and filamentary structure of density can also be explained by the interaction of the IAW with various other modes of waves present in the Earth's auroral region such as fast magnetosonic, slow magnetosonic, low-frequency IAW, and ion acoustic waves. Frycz et al. [28] investigated the nonlinear interaction of the dispersive shear Alfvén wave and ion acoustic wave in the Earth's magnetosphere considering two limits in their model: low- $\beta$ plasma (finite electron inertia) and high- $\beta$ plasma (electron thermal effect). Their results suggest that the ponderomotive force steepens the SAW leading to rapid density and electric field fluctuations. Mottez [29] also extended his investigation about the interaction of parallel propagating Alfven waves to explain the formation of deep plasma cavities in the Earth auroral zone.

In the present paper, we propose to study the nonlinear coupling between IAWs and MSWs taking the correction effect of finite frequency $\left(\omega_{0}<\omega_{c i}\right)$ of the pump IAW applicable to auroral plasma. This leads to a change in the dispersive properties of IAWs and also in the resulting localized structures, turbulence scaling, and density depletions (cavities). From the observation of Hawkeye 1, Kintner [30] showed that the average turbulence scaling near the auroral region varies as $4.02 \pm 0.59$ at the smaller scales. A similar observation is recorded by Heos 2 spacecraft, as reported by D'Angelo et al. [31] for the polar cusp region. Gurnett et al. [32] also reported similar scaling from the observation of Dynamics Explorer 1. Small-scale Alfvénic structures are also observed in aurora and are considered to be associated with stochastic heating of the plasma particles in the auroral region $[19,33]$.

Although the localization of the IAW and density cavitations have been discussed in the literature, nonlinear interaction of the 3D-IAW including correction effect of finite frequency $\left(\omega_{0}<\omega_{c i}\right)$ of the pump IAW with the perpendicularly propagating magnetosonic wave (PMSW) has not been studied in detail. The main aim of this work is to study the nonlinear coupling of finite-frequency IAW (when the frequency of the IAW is not much less than the ion gyrofrequency $\left(\omega_{0}<\omega_{c i}\right)$ ) with the PMSW to study the formation of localized structures, turbulence, and density cavitations for the auroral region. It should be pointed out here that recently, observation of the magnetosonic wave has been reported by THEMIS [34] in the Earth's magnetosphere. For this purpose, the coupled system of 3D-IAW and PMSW, in the presence of ponderomotive nonlinearity, has been developed using a two-fluid approach. Furthermore, numerical simulation has been pointed out to study the nonlinear stage of modulation instability. The content of the paper is organized as follows: dynamics of the 3D-IAW and PMSW has been presented in Sections 2 and 3, respectively. Numerical simulation and results are presented in Sections 4 and 5, respectively. Results are discussed in Section 5, and finally, Section 6 comprises the conclusion.

\section{Dynamics of the Inertial Alfvén Wave}

The nonlinear governing equation of the finite-frequency $\left(\omega_{0}<\omega_{c i}\right)$ IAW is assumed to be propagating in the $x-y-$ $z$ plane, i.e., $\vec{k}=k_{x} \hat{x}+k_{y} \hat{y}+k_{z} \widehat{z}$ having background magnetic field $B_{0}$ along the $z$-direction, i.e., $\vec{B}_{0}=B_{0} \widehat{z}$, and the dynamical equation for the $3 \mathrm{D}$-IAW can be obtained as follows:

$$
\begin{aligned}
\frac{\partial^{2} \widetilde{A}_{z}}{\partial t^{2}}= & \lambda_{e}^{2}\left(\frac{\partial^{4} \widetilde{A}_{z}}{\partial t^{2} \partial x^{2}}+\frac{\partial^{4} \widetilde{A}_{z}}{\partial t^{2} \partial y^{2}}\right)+\frac{V_{A}^{2}}{\omega_{c i}^{2}}\left(\omega_{c i}^{2}+\frac{\partial^{2}}{\partial t^{2}}\right) \\
& \cdot\left(1-\frac{n}{n_{0}}\right) \frac{\partial^{2} \widetilde{A}_{z}}{\partial z^{2}}
\end{aligned}
$$

Here, $n \approx \tilde{n}_{e} \approx \tilde{n}_{i}$ and $\lambda_{e}=c / \omega_{p e}$ is the electron inertial length. Equation (1) gives the dispersion relation as follows:

$$
\frac{\omega_{0}{ }^{2}}{{V_{A}}^{2}{k_{0 z}}^{2}}=\frac{1}{\left\{1+\lambda_{e}{ }^{2}\left({k_{0 x}}^{2}+{k_{0 y}}^{2}\right)+\lambda_{i}{ }^{2}{k_{0 z}}^{2}\right\}}
$$

Here, $\lambda_{i}=c / \omega_{p i}$ is an ion inertial length. The term $\lambda_{i}{ }^{2} k_{0 z}{ }^{2}$ in equation (2) appears due to the finite frequency correction $\left(\omega_{0} / \omega_{c i}\right)$.

We seek the solution of equation (1) for vector potential $A_{z}$ as follows: 


$$
\widetilde{A}_{z}=A_{z}(x, y, z, t) e^{i\left(k_{0 x} x+k_{0 y} y+k_{0 z} z-\omega_{0} t\right)} .
$$

Using equation (3) in equation (1), the following equation has been obtained for the case when $\partial_{z} A_{z} \ll k_{0 z} A_{z}$,

$$
\begin{gathered}
i \frac{2 \omega_{0}\left\{1+\lambda_{e}{ }^{2} k_{0 \perp}{ }^{2}+\tau\right\}}{V_{A}{ }^{2} k_{0 z}{ }^{2}(1-\alpha)} \frac{\partial A_{z}}{\partial t}-2 i k_{0 \perp} \frac{\omega_{0}{ }^{2} \lambda_{e}{ }^{2}}{V_{A}{ }^{2} k_{0 z}{ }^{2}(1-\alpha)}\left(\frac{\partial A_{z}}{\partial x}+\frac{\partial A_{z}}{\partial y}\right) \\
-\frac{\omega_{0}{ }^{2} \lambda_{e}{ }^{2}}{V_{A}{ }^{2} k_{0 z}{ }^{2}(1-\alpha)}\left(\frac{\partial^{2} A_{z}}{\partial x^{2}}+\frac{\partial^{2} A_{z}}{\partial y^{2}}\right)+i \frac{2}{k_{0 z}} \frac{\partial A_{z}}{\partial z}+\frac{n}{n_{0}} A_{z}=0 .
\end{gathered}
$$

Here, $\alpha=\omega_{0}^{2} / \omega_{c i}^{2}, \tau=V_{A}{ }^{2} k_{0 z}^{2} / \omega_{c i}^{2}, k_{0 x}, k_{0 y}\left(k_{0 z}\right)$ is the component of the wave vector perpendicular (parallel) to $B_{0} \widehat{z}$, and $\omega_{0}$ is the frequency of the 3D-IAW.

\section{Dynamics of the Magnetosonic Wave}

Let us assume that the low-frequency magnetosonic wave is propagating perpendicularly with respect to the background magnetic field along the $x$ - axis, i.e., $\vec{k}=k_{x} \widehat{x}$. The dynamical equation for the PMSW can be obtained by the standard approach using the basic plasma equations mentioned in the following:

(i) The equation of motion:

$$
\frac{\partial \vec{v}_{j}}{\partial t}=\frac{q_{j}}{m_{j}} \vec{E}+\frac{q_{j}}{c m_{j}}\left(\vec{v}_{j} \times \vec{B}_{0}\right)-\frac{\gamma_{j} k T}{m_{j}} \vec{\nabla} \frac{n_{j}}{n_{0}}+\vec{F}_{j}
$$

(ii) The continuity equation:

$$
\frac{\partial n}{\partial t}+\vec{\nabla} \cdot\left(n \vec{v}_{j}\right)=0
$$

(iii) Faraday's law:

$$
(\vec{\nabla} \times \vec{E})=-\frac{1}{c} \frac{\partial \vec{B}}{\partial t}
$$

Here, the index $j=e$ or $i$ accounts for the electrons and ions, respectively, $q_{i}=-q_{e}=e$ is the charge, $j=e n_{0}\left(v_{\mathrm{i}}-v_{\mathrm{e}}\right)$ is the current density, $v_{j}$ is the velocity, $n_{0}$ is the background number density, $m_{j}$ and $T_{j}$ are the masses and temperature of $j$ th species, and $\vec{F}_{j}=-\left[m_{j}\left(\vec{v}_{j} \cdot \vec{\nabla}\right) \vec{v}_{j}-q_{j} / c\left(\vec{v}_{j} \times \vec{B}_{0}\right)\right]$ is the ponderomotive force due to 3D-IAW. Putting the values of $\vec{v}_{j}$ in the wave equation and taking the $y$ component of that, one can have

$$
\begin{aligned}
\frac{\partial^{2} E_{y}}{\partial x^{2}}-\frac{1}{c^{2}} \frac{\partial^{2} E_{y}}{\partial t^{2}}-\frac{1}{v_{A}^{2}} \frac{\partial^{2} E_{y}}{\partial t^{2}}= & \frac{4 \pi n_{0} T_{e}}{c B_{0}} \frac{\partial^{2}}{\partial x \partial t}\left(\frac{n_{e}}{n_{0}}\right) \\
& +\frac{4 \pi n_{0} e}{c^{2}}\left[-\frac{\omega}{i \omega_{c j}} \frac{F_{j x}}{m_{j}}-\frac{\omega^{2}}{i \omega_{c j}^{2}} \frac{F_{j y}}{m_{j}}\right] .
\end{aligned}
$$

Here, ions are assumed to be cold and $\omega \ll \omega_{c i}$. The electron continuity equation yields

$$
\frac{\partial}{\partial t}\left(\frac{n_{e}}{n_{0}}\right)=-\frac{\partial}{\partial x}\left(\frac{c E_{y}}{B_{0}}\right)
$$

Components of ponderomotive force are given as

$$
F_{e x}=\left(\frac{\omega_{o}}{B_{0}}\right)^{2}\left[-\frac{m_{e}}{4} \frac{(1+\varepsilon)^{2} k_{\perp 0}^{2}}{2 k_{z 0}^{2}}+\frac{m_{i}}{8} \frac{(1+\varepsilon) k_{\perp 0}^{2}}{(1-\alpha) k_{z 0}^{2}}\right] \frac{\partial}{\partial x}\left|A_{0 z}\right|^{2},
$$

$F_{e y}=\frac{m_{e}}{4}\left(\frac{\omega_{0}}{B_{0}}\right)^{2} \frac{(1+\varepsilon)^{2} k_{\perp 0}^{2}}{2 k_{z 0}^{2}} \frac{\partial}{\partial x}\left|A_{0 z}\right|^{2}$,

$$
F_{i x}=\frac{m_{i}}{4}\left(\frac{\omega_{o}}{B_{0}}\right)^{2}\left[-\frac{(1+\varepsilon)^{2}(1+\alpha) k_{\perp 0}^{2}}{(1-\alpha)^{2} k_{z 0}^{2}}+\frac{\varepsilon}{\alpha}\right] \frac{\partial}{\partial x}\left|A_{0 z}\right|^{2},
$$

$$
F_{i y}=\frac{m_{i}}{4}\left(\frac{\omega_{o}}{B_{0}}\right)^{2}\left[\frac{(1+\varepsilon)^{2} k_{\perp 0}^{2}}{2(1-\alpha)^{2} k_{z 0}^{2}}\left\{1+\left(\frac{2 i}{1-\alpha}\right)\left(\frac{\omega_{0}}{\omega_{c i}}\right)\right\}\right] \frac{\partial}{\partial x}\left|A_{0 z}\right|^{2}
$$

Substituting equations (9), (11), and (12) into equation (8), one obtains

$$
\begin{aligned}
& \left((1+\beta) \frac{\partial^{2}}{\partial x^{2}}-\frac{1}{V_{A}{ }^{2}} \frac{\partial^{2}}{\partial t^{2}}\right)\left(\frac{n_{e}}{n_{0}}\right)= \\
& \frac{\omega_{0}^{2}}{4 V_{A}{ }^{2} B_{0}{ }^{2}}\left\{-\frac{\left\{(1+\varepsilon)^{2} k_{0 \perp}{ }^{2}\right\}}{2 k_{0 z}^{2}}\left\{\frac{(1+\alpha)}{(1-\alpha)^{2}}+\frac{m_{e}}{m_{i}}\right\}+\left\{\frac{\varepsilon}{\alpha}+\frac{k_{0 \perp}{ }^{2}}{k_{0 z}^{2}}\right\}\right\}\left(\frac{\partial^{2}\left(\left|A_{z}\right|^{2}\right)}{\partial x^{2}}\right) .
\end{aligned}
$$


Here, $k_{0 \perp}{ }^{2}=k_{0 x}{ }^{2}+k_{0 y}{ }^{2}, \beta=C_{s}^{2} / V_{A}^{2}, \varepsilon=k_{0 \perp}{ }^{2} \lambda_{e}{ }^{2}$, and $v_{A}=\left(B_{0}^{2} / 4 \pi n_{0} m_{i}\right)^{1 / 2}$.

Equation (13) represents the dynamical equation of the PMSW, and its right-hand side represents the ponderomotive force due to 3D-IAW.

After normalization of equations (4) and (13), these can be written in the dimensionless form as

$$
i \frac{\partial A_{z}}{\partial t}-i \xi_{1} \frac{\partial A_{z}}{\partial x}-\xi_{1} \frac{\partial A_{z}}{\partial y}-\xi_{2} \frac{\partial^{2} A_{z}}{\partial x^{2}}-\xi_{2} \frac{\partial^{2} A_{z}}{\partial y^{2}}+i \frac{\partial A_{z}}{\partial z}+n A_{z}=0
$$

$$
\left(\frac{\partial^{2}}{\partial x^{2}}-\xi_{3} \frac{\partial^{2}}{\partial t^{2}}\right) n=-\frac{\partial^{2}\left(\left|A_{z}\right|^{2}\right)}{\partial x^{2}}
$$

Here, $\quad \xi_{1}=2 \omega_{0}{ }^{2} / V_{A}{ }^{2} k_{0 z}{ }^{2} k_{0 \perp} \lambda_{e}(1-\alpha), \quad \xi_{2}=\omega_{0}^{2} /$ $V_{A}{ }^{2} k_{0 \perp}{ }^{2} \lambda_{e}{ }^{2} k_{0 z}{ }^{2}(1-\alpha), \quad$ and $\xi_{3} \approx V_{A}^{2} \lambda_{e}^{2} / 4(1+\beta)\left\{k_{0 z}^{2}(1-\right.$ $\left.\alpha) / \omega_{0}\left(1+\lambda_{e}^{2} k_{0 \perp}^{2}+\tau\right)\right\}^{2}$. The normalizing parameters are $x_{n} \approx y_{n} \approx \quad \lambda_{e}, z_{n} \approx 1 / 2 k_{0 \perp}^{2} \lambda_{e}^{2} k_{0 z}, t_{n} \approx 2 \omega_{0}\{1+$ $\left.\lambda_{e}{ }^{2} k_{0 \perp}^{2}+\tau\right\} / V_{A}{ }^{2} k_{0 z}^{2}(1-\alpha), n_{n} \approx k_{0 \perp}{ }^{2} \lambda_{e}{ }^{2} n_{0}, \quad$ and $\left(A_{z}\right)_{n} \approx$ $\left(4 \lambda_{e}^{2} V_{A}^{2}(1+\beta) / \omega_{0}^{2}\left\{-\left\{(1+\varepsilon)^{2}\left(k_{0 x}^{2}+\quad k_{0 y}^{2}\right)\right\} / 2 k_{0 z}^{2}\right\}\{(1+\right.$ $\left.\left.\alpha) /(1-\alpha)^{2}+m_{e} / m_{i}\right\}+\left\{\varepsilon / \alpha+\left(k_{0 x}^{2}+k_{0 y}^{2}\right) / k_{0 z}^{2}\right\}\right)^{1 / 2} B_{0}$.

\section{Numerical Simulation}

Numerical simulation has been performed using the pseudospectral method for equations (13) and (14). The wave numbers of perturbation are taken as $\alpha_{x}=\alpha_{y}=\alpha_{z}=0.2$ which is normalized by $x_{n}^{-1}, y_{n}^{-1}$, and $z_{n}^{-1}$, respectively. Simulation has been carried out with $(64)^{3}$ grid points, and a periodic spatial domain of $\left(2 \pi / \alpha_{x}\right) \times\left(2 \pi / \alpha_{y}\right) \times\left(2 \pi / \alpha_{z}\right)$ with the initial conditions of simulation is as follows:

$$
\begin{aligned}
A_{z}(x, y, z, 0)= & A_{z 0}\left(1+0.1 \cos \left(\alpha_{x} x\right)\right)\left(1+0.1 \cos \left(\alpha_{y} y\right)\right) \\
& \cdot\left(1+0.1 \cos \left(\alpha_{z} z\right)\right),
\end{aligned}
$$

$$
n(x, y, z, 0)=-\left|A_{z}(x, y, z, 0)\right|^{2}
$$

Here, $\left|A_{z 0}\right|=1$ is the amplitude of the homogenous pump 3D-IAW. An algorithm for the nonlinear Schrödinger (NLS) equation has been studied and tested against its invariant in order to carry out the simulation of equations (13) and (14).

The accuracy was determined by consistency of the number $N=\sum_{k}\left|A_{z k}\right|^{2}$ in the case of cubic NLS equation. During computation, the conserved quantity was preserved to the order of $10^{-5}$. After testing algorithm of cubic NLS, it has been modified for solving dimensionless equations (13) and (14), which is used to study nonlinear coupling of 3DIAW with PMSW and the resulting formation of localized structures, turbulent scaling, and density cavities.

The values of $\xi_{1}$ and $\xi_{2}$ can be evaluated from the low- $\beta$ plasma parameters applicable to aurora. For the application purpose in low- $\beta$ plasma, the typical parameters for the auroral altitude of $1700 \mathrm{~km}$ [11] are as follows: $B_{0} \approx 0.3 G, n_{0} \approx 5 \times 10^{3} \mathrm{~cm}^{-3}$, and $T_{e} \approx 1.16 \times 10^{4} \mathrm{~K}$. Using these parameters, one can find $V_{A} \approx 9.25 \times 10^{8} \mathrm{~cm} / \mathrm{s}, \lambda_{e} \approx$
$7.52 \times 10^{3} \mathrm{~cm}, \omega_{c i} \approx 2.87 \times 10^{3} \mathrm{sec}^{-1}, \rho_{s} \approx 481.80 \mathrm{~cm}$, and $c_{s}=1.38 \times 10^{6} \mathrm{~cm} / \mathrm{s}$. For $k_{0 \perp} \lambda_{e} \approx 0.30$ and $\omega_{0} / \omega_{c i} \approx 0.30$, one can calculate $k_{0 x} \approx 1.19 \times 10^{-5} \mathrm{~cm}^{-1}, k_{0 y} \approx 1.19 \times 10^{-}$ $5 \mathrm{~cm}^{-1}, k_{0 z} \approx 6.38 \times 10^{-7} \mathrm{~cm}^{-1}$, and $\omega_{0} \approx 0.574 \times 10^{3} \mathrm{sec}^{-1}$. The normalizing parameter values are $x_{n} \approx 7.52 \times 10^{3} \mathrm{~cm}, y_{n} \approx 7.52 \times 10^{3} \mathrm{~cm}, z_{n} \approx 9.66 \times 10^{7} \mathrm{~cm}$, $t_{n} \approx 0.11 \mathrm{sec}, n_{n} \approx 0.16 \times 10^{3} \mathrm{~cm}^{-3}$, and $\left(A_{z}\right)_{n} \approx 5.36 \times$ $10^{3} \mathrm{G}-\mathrm{cm}$.

\section{Results and Discussion}

In Section 4, numerical simulation has been carried out for dimensionless equations (13) and (14) to study the formation of localized structures, turbulent scaling, and density cavitation phenomenon, applicable to the auroral region. In simulation, time history of nonlinear evolution is studied from state $t=0$ to the state when the system reaches to the quasi-steady state. For the chosen parameters, the time required to set up the ponderomotive nonlinearity $r_{0} /$ $c_{\mathrm{s}} \sim 0.2 \mathrm{sec}$. In order to study the effect of ponderomotive nonlinearity, simulation is carried out from $t=0$ to the time $t=29(\sim 3.22 \mathrm{sec})$, which is larger than the time required to set up the ponderomotive nonlinearity and to reach the system in the quasi-steady state. Results shown in Figures 1-3 are obtained at different time scales after the system achieves the quasi-steady state. Equation (12) represents the dynamical equation of the PMSW, and its righthand side represents the ponderomotive force due to $3 \mathrm{D}$ IAW, and in equation (15), the time-dependent density depends on magnitude of ponderomotive force as well as the interaction between 3D-IAW and PMSW. Figures 1(a)-1(c) show the magnetic field intensity profile of the IAW in the $X$ $Y$ plane at time $t=18,24$, and 29, respectively, showing a nonlinear evolution of the coherent structure, while Figure 1(d) presents the evolution of Fourier transformation $\left|A_{k z}\right|^{2}$ of the IAW with $K_{x}-K_{y}$ at time $t=22$. One can observe from Figures $1(\mathrm{a})-1(\mathrm{~d})$ that the magnetic field intensity gets localized and becomes more complex with time. The PMSW in the presence of the nonlinearity (ponderomotive force, which changes with time) starts getting localized and hence affects the dynamics of the 3D-IAW which leads to the localization of the 3D-IAWs through equations (13) and (14).

Next, the power spectral index has been illustrated in Figure 2. It reveals the variation of $\left|A_{z k}\right|^{2}$ against $k$ at time $t=29$. It is evident from the wavenumber spectrum that, for $k \lambda_{e}<1$, the spectral index follows nearly Kolmogorov $\left(\sim k^{-5 / 3}\right)$ scaling (a typical inertial range scaling). A spectral break appears at $k \lambda_{e} \approx 1$, and for $k \lambda_{e}>1$, the spectral index follows nearly $\left(\sim k^{-4.28}\right)$. From the steepening of the spectrum, it is clear that the nonlinear interaction of the $3 \mathrm{D}$ IAW and PMSW might be helpful for the energy transfer from a small wavenumber to large wavenumber. These kinds of power spectra have also been reported (observation of Hoes 2, Hawkeye 1, and Dynamics Explorer 1 spacecrafts) in the auroral and other magnetosphere regions by D'Angelo et al. [31], Kintner [30], and Gurnett et al. [32].

Figures 3(a)-3(c) represent the 3D evolution of the resulting density structures in the $x-y$ plane. From Figure 3, one can observe that three-dimensional (3D) density dips are 


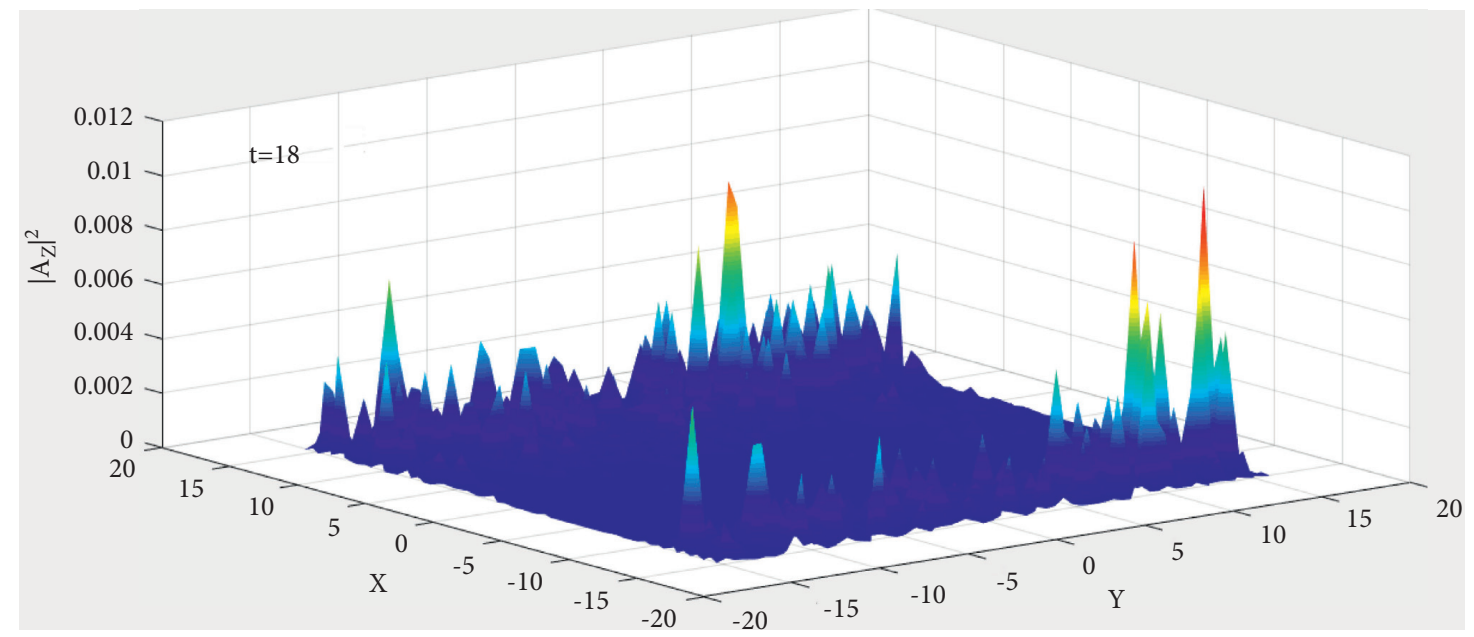

(a)

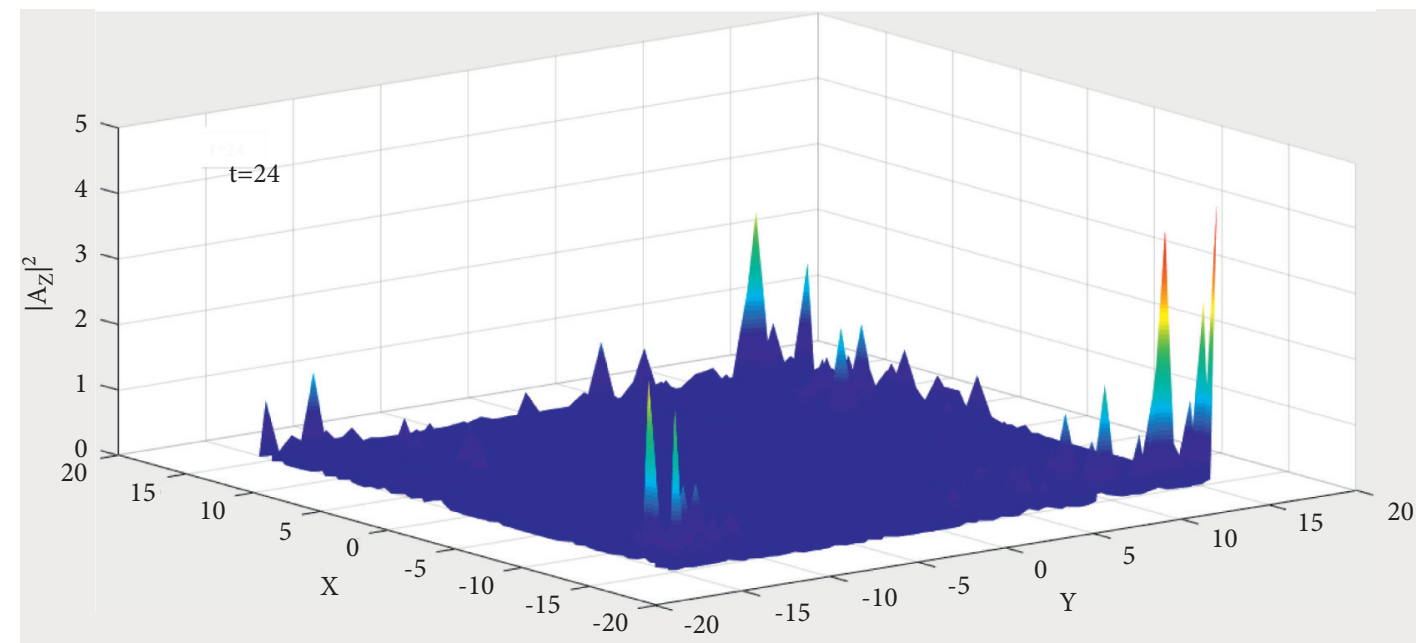

(b)

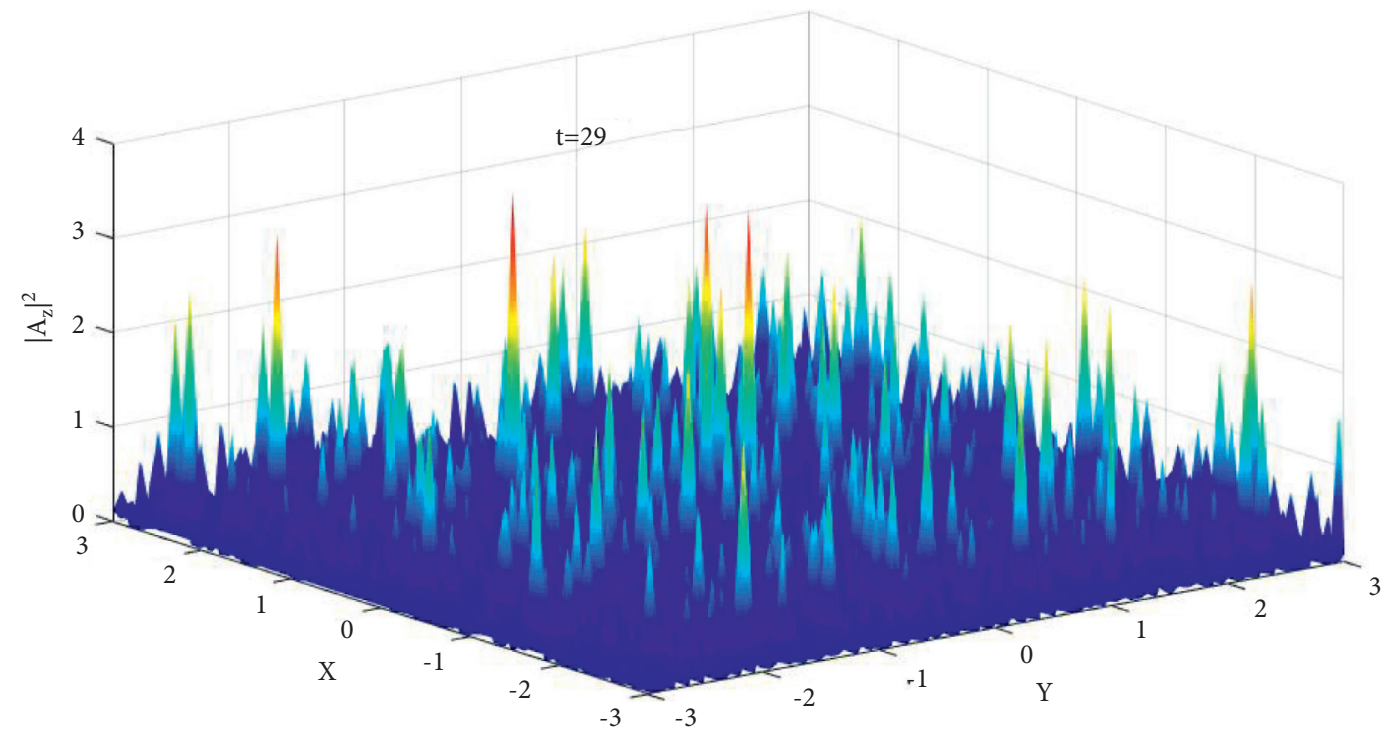

(c)

Figure 1: Continued. 


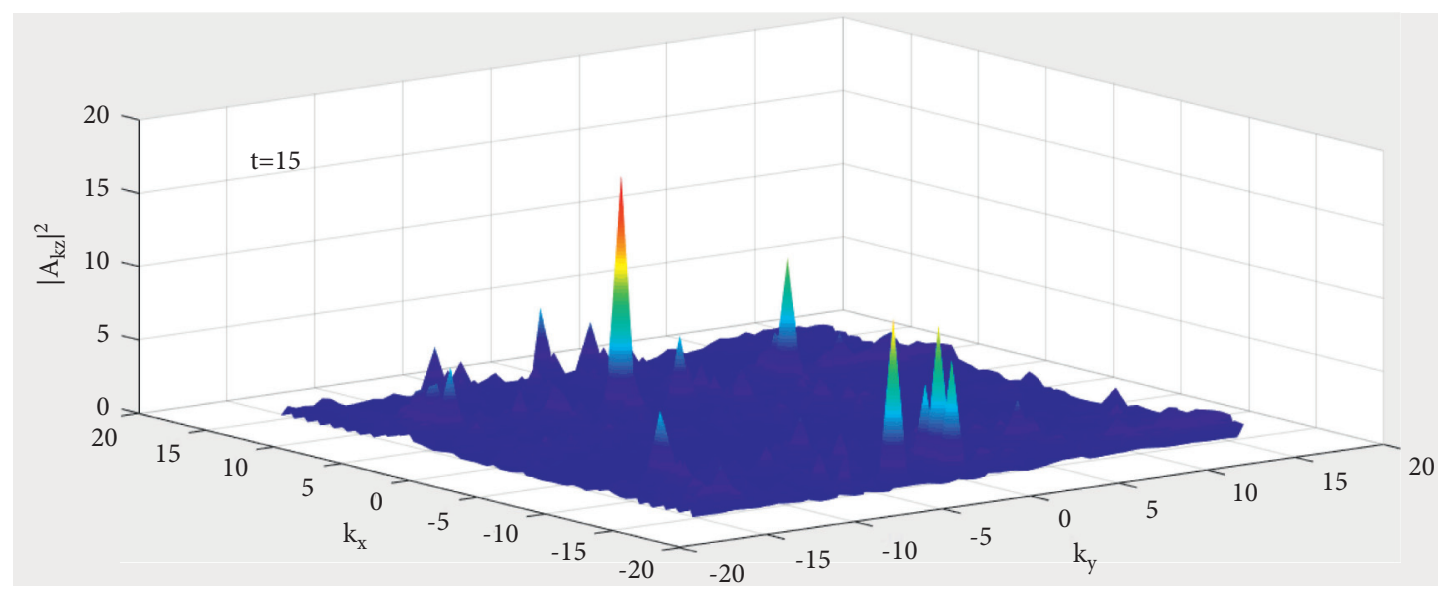

(d)

Figure 1: (a) The magnetic field intensity profile of the IAW for low- $\beta$ plasma (auroral region) at time $t=18$. (b) The magnetic field intensity profile of the IAW for low- $\beta$ plasma (auroral region) at time $t=24$. (c) The magnetic field intensity profile of the IAW for low- $\beta$ plasma (auroral region) at time $t=29$. (d) Evolution of $\left|A_{k z}\right|^{2}$ of the IAW with $K_{x}-K_{y}$ at time $t=22$.

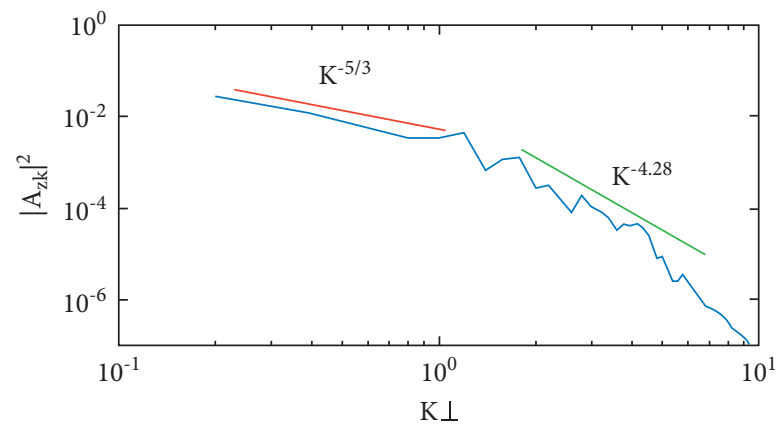

FIGURE 2: Variation of $\left|A_{z k}\right|^{2}$ (power spectrum) against $k$ for low- $\beta$ plasma (auroral region) at time $t=29$.

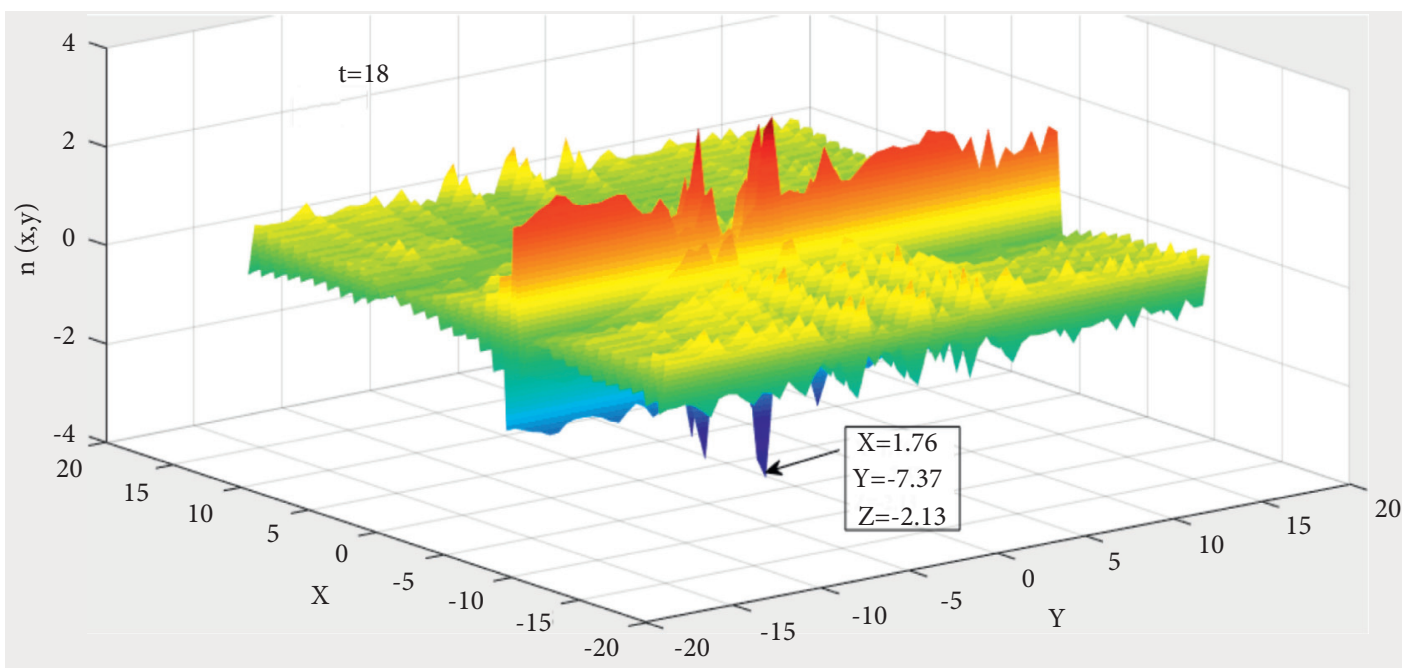

(a)

Figure 3: Continued. 


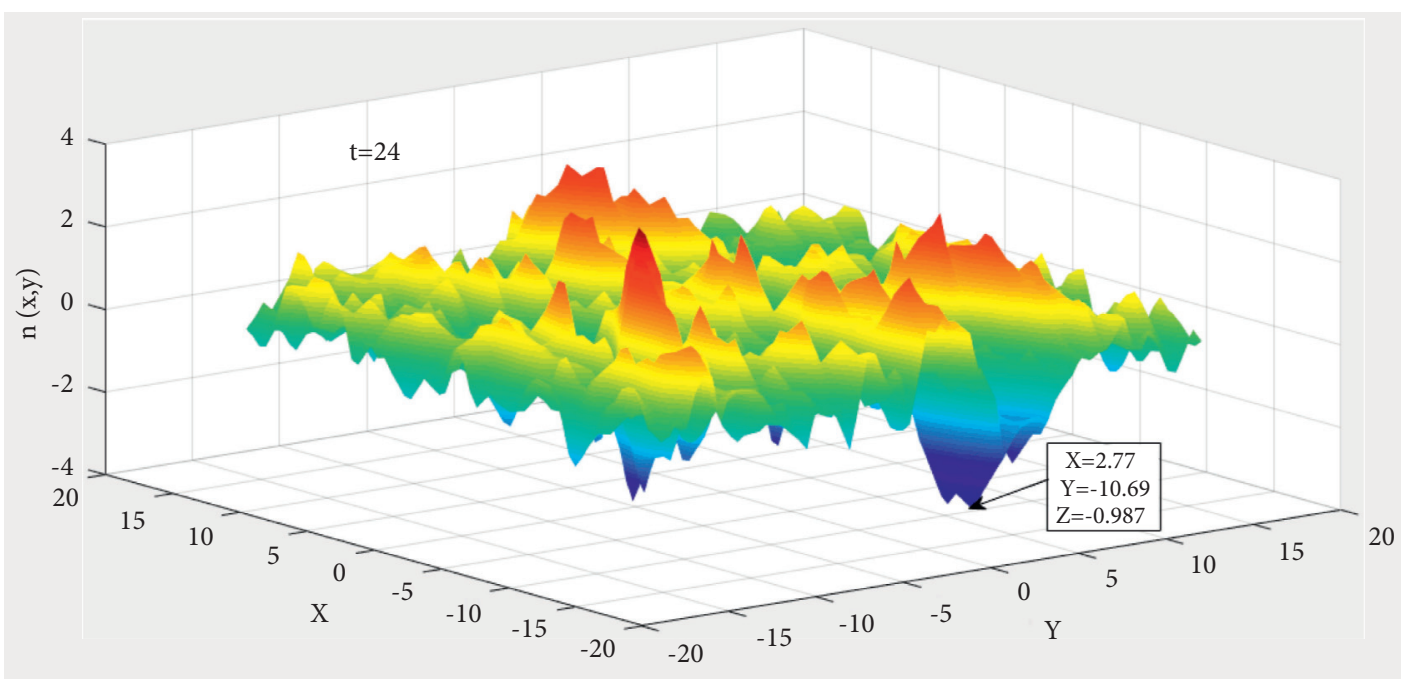

(b)

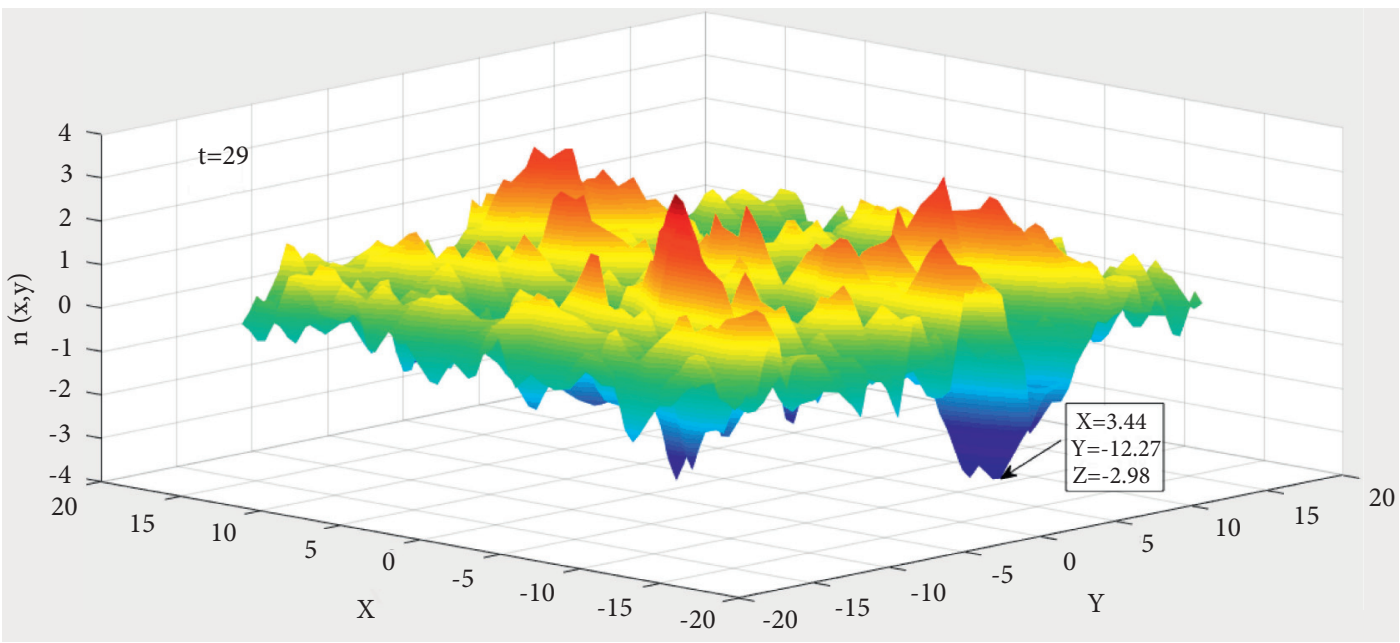

(c)

Figure 3: (a) 3D evolution of the density profile at time $t=18$. (b) $3 \mathrm{D}$ evolution of the density profile at time $t=24$. (c) $3 \mathrm{D}$ evolution of the density profile at time $t=29$.

formed which changes with time. The formation of the coherent structure and turbulence in density profile can be explained with the help of equations (13) and (16). Equation (13) represents the dynamical equation of the PMSW, and its right-hand side represents the ponderomotive force due to 3D-IAW where the intensity of the IAW is $X-Y$ dependent. At the same time, equation (15) represents that the density profile of the PMSW also depends on amplitude of the pump 3D-IAW. Although the PMSW is propagating along the $X$-direction, the ponderomotive force due to 3D-IAW and the interaction between the 3D-IAW and PMSW alter the density of the PMSW in $X-, Y$-, and $Z$ directions. Since the interaction of the IAW and PMSW is chaotic at later times, not only the location of density dips changes but also the depth of these dips changes with time (marked as $x, y$, and $z$ in Figure 3). The nonlinear interaction between IAW and PMSW varies in space with time, and hence, the magnitude of ponderomotive force associated with them also changes in space with time accordingly. Eventually the density profile is affected by the ponderomotive force and creates the density cavity of different depth at different position with time. The magnetic field is trapped in the regions of low density due to the ponderomotive nonlinearity. Small-scale length density cavities have been observed in the auroral zone by Viking and Freja spacecrafts $[35,36]$. For the auroral region, we observed the density fluctuations of $\sim 0.12 n_{0}$, consistent with the FAST observation reported by Chaston et al. $[14,15]$. Thus, in the present paper, efforts have been made to understand the phenomena of turbulence, localization, and density cavitations due to the nonlinear interplay of the finite-frequency IAW $\left(\omega_{0}<\omega_{c i}\right)$ with the PMSW. The finite frequency correction effect is expected to change the dispersive property of the 3D-IAW and resulting ponderomotive force by the IAW.

\section{Conclusion}

To summarize, our primary aim of the present study is to understand the physical mechanism behind the processes of localization and density cavitations during the 
nonlinear interaction of the finite-frequency IAW $\left(\omega_{0}\right.$ $\left.<\omega_{c i}\right)$ with the PMSW. Numerical simulation of equations (13) and (14), governing the dynamics of the 3D-IAW and PMSW, has been carried out, applicable to the auroral region. The background density of the PMSW gets altered due to the ponderomotive nonlinearity of the pump 3DIAW, which results in the density cavitations and field localization. The field associated with the 3D-IAW gets trapped in the density cavities and breaks into a localized coherent structure. The result reveals that these localized structures and density cavity become progressively more complex and grow towards larger to smaller length scales. The depth of the density cavity and formation of the localized structure depend on the magnitude of the ponderomotive force and the nature of the interacting waves. From the obtained results, we have found that the depth of density cavities is of the order of $\sim 0.10 n_{0}$, and these density-depleted regions are of the transverse scale size of the order of electron inertial length. These kinds of small-scale-sized density cavities have been observed in the auroral region and are consistent with the FAST satellite observation reported by Chaston et al. $[14,15]$.

Since the nature of the interacting waves discussed in the present paper is different at various locations, the magnitude of ponderomotive force associated with them is also different. This is the main reason that the depth of the density cavity in each location is not the same. Therefore, it becomes clear that the depth of the density cavities changes with the magnitude of the ponderomotive force and the nature of the waves associated with them. On the basis of obtained results, we can conclude that the nonlinear interaction of the 3D-IAW and PMSW is responsible for the localized coherent structure and density cavitations.

\section{Data Availability}

The main aim of this work is to study the nonlinear coupling of the finite-frequency IAW (when the frequency of the IAW is not much less than the ion gyrofrequency) with the PMSW to study the formation of localized structures, turbulence, and density cavitation for the auroral region. It should be pointed out here that recently, the observation of the magnetosonic wave has been reported by THEMIS [34] in the Earth's magnetosphere.

\section{Conflicts of Interest}

The authors declare that they have no conflicts of interest.

\section{Authors' Contributions}

Prof. R. P. Sharma proposed the problem, and under his guidance, Motilal Rinawa and Prashant Chauhan formulated the analytical part, while Sintu Kumar was responsible for numerical simulation and result preparation. Manoh Kumar Singh and Hari Kumar Singh were responsible to simulate the results in the revised manuscript, while Amit Sharma helped in the preparation of the revised manuscript.

\section{Acknowledgments}

This research was partially supported by the Indian Space Research Organisation (ISRO) under the RESPOND Programme and Department of Science and Technology (DST), India.

\section{References}

[1] W. Bostick and M. Levine, "Experimental demonstration in the laboratory of the existence of magneto-hydrodynamic waves in ionized helium," Physical Review, vol. 94, p. 815, 1952.

[2] H. Alfvén, "Existence of electromagnetic-hydrodynamic waves," Nature, vol. 150, no. 3805, pp. 405-406, 1942.

[3] W. Gekelman, "Review of laboratory experiments on Alfvén waves and their relationship to space observations," Journal of Geophysical Research, vol. 104, p. 14417, 1999.

[4] P. K. Shukla, L. Stenflo, T. Passot, and P. L Sulem, Nonlinear Phenomena Involving Dispersive Alfvén Waves, SpringerVerlag, vol. 1, p. 30, Berlin, Heildelberg, 1999.

[5] C. K. Goertz and R. W. Boswell, "Magnetosphere-ionosphere coupling," Journal of Geophysical Research, vol. 84, p. 7239, 1979.

[6] J. V. Hollweg, "Kinetic Alfvén wave revisited," Journal of Geophysical Research, vol. 104, no. 14, pp. 811-814, 1999.

[7] C. C. Chaston, "Energy deposition by Alfve' $n$ waves into the dayside auroral oval: cluster and FAST observations," Journal of Geophysical Research, vol. 110, 2005.

[8] J. R. Wygant, A. Keiling, C. A. Cattell et al., "Evidence for kinetic Alfvén waves and parallel electron energization at 4-6 RE altitudes in the plasma sheet boundary layer," Journal of Geophysical Research, vol. 107, p. 1201, 2002.

[9] C. S. Wu, C. B. Wang, and Q. M. Lu, "Density depletion in a coronal flux tube associated with solar radio emission," Solar Physics, vol. 235, p. 317, 2006.

[10] D. J. Wu and J. K. Chao, "Model of auroral electron acceleration by dissipative nonlinear inertial Alfvén wave," Journal of Geophysical Research, vol. 109, p. A06211, 2004.

[11] D. J. Wu, G. L. Huang, and D. Y Wang, "Dipole density solitons and solitary dipole vortices in an inhomogeneous space plasma," Physical Review Letters, vol. 77, pp. 4346-4349, 1996.

[12] P. M. Bellan and K. Stasiewicz, "Fine-scale cavitation of ionospheric plasma caused by inertial Alfvén wave ponderomotive force," Physical Review Letters, vol. 80, no. 16, pp. 3523-3526, 1998.

[13] R. F. Benson and A. Hilgers, "Comment on "The auroral radiating plasma cavities" by A. Hilgers," Geophysical Research Letters, vol. 22, no. 21, pp. 3005-3007, 1995.

[14] C. C. Chaston, C. W. Carlson, R. E. Ergun, and J. P. McFadden, "Alfvén waves, density cavities and electron acceleration observed from the FAST spacecraft," Physica Scripta, vol. T84, p. 64, 2000.

[15] C. C. Chaston, C. W. Carlson, J. P. McFadden, and R. J. Strangeway, "How important are dispersive Alfve'n waves for auroral particle acceleration?" Geophysical Research Letters, vol. 34, p. L07101, 2007.

[16] E. M. Dubinin, P. L. Israelevich, I. Kutiev, and I. M. NikolaevaNSAnd Podgorny, "Localized auroral disturbance in the morning sector of topside ionosphere as a standing electromagnetic wave," Planetary and Space Science, vol. 33, pp. 597-606, 1985. 
[17] K. Stasiewicz, G. Holmgren, and L. Zanetti, "Density depletions and current singularities observed by Freja," Journal of Geophysical Research, vol. 103, no. A3, p. 4251, 1998.

[18] A. Hasegawa and L. Chen, "Parametric decay of kinetic Alfvén wave and its application to plasma heating," Physical Review Letters, vol. 36, p. 1362, 1976.

[19] K. Stasiewicz, P. Bellan, C. Chaston et al., "Small scale Alfvenic structure in the aurora," Space Science Reviews, vol. 92, pp. 423-533, 2000.

[20] B. Buti, M. Velli, and P. C. Liewer, B. E. Goldstein, , and Hada, T. Hybrid simulations of collapse of Alfvénic wave packets," Plasmas, vol. 7, p. 3998, 2000.

[21] M. L. Goldstein and D. A. Roberts, "Magneto hydrodynamic turbulence in the solar wind," Physical Plasmas, vol. 6, p. 4154, 1999.

[22] G. G. Howes, S. C. Cowley, and W. Dorland, "A model of turbulence in magnetized plasmas: implications for the dissipation range in the solar wind," Journal of Geophysical Research, vol. 113, p. A05103, 2008.

[23] J. S. Zhao, D. J. Wu, and J. Y Lu, "Kinetic Alfvén turbulence and parallel electric fields in flare loops," ApJ, vol. 767, p. 109, 2013.

[24] D. Sundkvist, V. Krasnoselskikh, P. K. Shukla et al., "In situ multi-satellite detection of coherent vortices as a manifestation of Alfvénic turbulence," Nature (London), vol. 436, pp. 825-828, 2005.

[25] J. S. Zhao, D. J. Wu, M. Y. Yu, and J. Y. Lu, "Genaration of convective cell by Kinetic Alfven waves," New Journal of Physics, vol. 13, Article ID 063043, 2011.

[26] J. S. Zhao, D. J. Wu, J. Y. LU, L. Yang, and M. Y. Yu, "Convective cell generation by Kinetic Alfven wave turbulence in auroral ionosphere," Physics of Plasmas, vol. 19, Article ID 062901, 2012.

[27] S. Kumar and H. D. Singh, "Cavitations by nonlinear interaction between inertial Alfvén waves and magneto sonic waves in low beta plasmas," Solar Physics, vol. 270, pp. 523-535, 2011.

[28] P. Frycz, R. Rankin, J. C. Samson, and V. T. Tikhonchuk, "Nonlinear field line resonances: Dispersive effects," Physics of Plasmas, p. 3565, 1998.

[29] F. Mottez, "Plasma acceleration by the interaction ofparallel propagating Alfv'en waves," Journal of Plasma Physics, p. 325810104, 2015.

[30] P. M. Kintner, "Observations of velocity shear driven plasma turbulence," Journal of Geophysical Research, vol. 81, p. 28, 1976.

[31] N. D'Angelo, A. Bahnsen, and H Rosenbauer, "Wave and particle measurements at the polar cusp," Journal of Geophysical Research, vol. 79, pp. 3129-3134, 1974.

[32] D. Gurnett, R. L. Huff, J. D. Menietti, J. L. Burch, J. D. Winningham, and S. D. Shawhan, "Correlated low-frequency electric and magnetic noise along the auroral field lines," Journal of Geophysical Research, vol. 89, pp. 8971-8985, 1984.

[33] J. S. Zhao, D. J. Wu, and J. Y. Lu, "Kinetic Alfvén waves excited by oblique magneto hydrodynamic Alfvén waves in coronal holes," Acta Pathologica Japonica, vol. 735, p. 114, $2011 \mathrm{~b}$.

[34] Q. Ma, W. Li, R. M. Thorne, and V. Angelopoulos, "Global distribution of equatorial magneto sonic waves observed by THEMIS," Geophysical Research Letters, vol. 40, pp. 18951901, 2013.

[35] C. C. Chaston, P. W. CarlsonCW, R. E. Ergun, and J. P. McFadden, "FASTObservations of inertial alfven waves in the dayside aurora," Geophysical Research Letters, vol. 26, pp. 647-650, 1999.

[36] E. Wahlund, P. Louarn, T. Chust et al., "On ion acoustic turbulence and the nonlinear evolution of kinetic Alfvén waves in aurora," Geophysical Research Letters, vol. 21, p. 1831, 1994. 